\title{
A Strain-Energy Model of Passive Knee Kinematics for the Study of Surgical Implantation Strategies
}

\author{
E. Chen, R.E. Ellis, and J.T. Bryant \\ Computing and Information Science Mechanical Engineering \\ Queen's University at Kingston, Ontario, Canada \\ Surgery \\ ellis@cs.queensu.ca
}

\begin{abstract}
A mathematical model for studying the passive kinematics of condylartype total knee prostheses can be useful in planning and performing total joint replacement. If the insertion location and neutral length of knee ligaments is known, the passive kinematics of the knee can be calculated by minimizing the strain energy stored in the ligaments in any angular configuration of the knee.

The model considered here takes into consideration the geometry of the prosthesis, patient-specific information, and operation-specific placement of the prosthesis. Based on an energy-minimization principle, this model can be used to study the kinematics of the knee joint of a patient after total joint replacement. The effect of various articular geometries, alternative surgical placements of prosthetic devices, and intraoperative ligamentous release can be simulated. The model may be useful in preoperative planning, intraoperative guidance, and the design of new prosthetic joints.
\end{abstract}

\section{Introduction}

One of the major goals of total knee replacement is to restore the normal function of the knee. The function of the knee after total joint replacement is heavily influenced by the design of the prosthesis and the surgical placement of the prosthetic devices [5]. In passive knee motion, where no external forces are present, the femur is kept in contact with the tibia by the tensile forces exerted by the surrounding ligaments. The geometry of the articular surfaces provides a set of feasible contact locations that determine the orientation of the knee. The interaction of the surrounding ligaments thus governs the contact conditions of the knee. It is supposed here that, at any given angulation, the contact condition that the knee would naturally assume is the contact condition that would minimize the total strain energy stored in the ligaments of the knee.

The theoretical goal of this work was to understand interactions between the geometry of the articular surfaces and the surrounding ligaments and to study how such interactions affect the overall kinematics of the knee. The passive kinematics were analyzed as the instantaneous quasi-static solution to ligament strain-energy minimization. This model required knowledge of the geometry of the articular surfaces, the insertion locations and mechanical properties of the knee ligaments, and the implanted location of the prosthetic components. The model determined the contact location between the femoral and tibial articular surfaces that minimized the strain energy stored in the ligaments of 
the knee. The ligament states, contact trajectories, and passive knee kinematics were analyzed to determine the effects of alternative surgical placement of the components. The knee kinematics were also animated, using standard computer graphics techniques, to provide a visualization of the passive motion of the knee.

The 3D mathematical model of the knee proposed in this paper is an extension of the 2D model proposed by Martelli et al.[6]. Based on the principle of energy minimization [4], the mathematical construction of our model is similar to the work by Wismans et al. [10] and Blankevoort et al. [1, 2, 7], but our model can be applied to other body joints with either prosthetic or natural articular surfaces. The motion studied is passive, which means no external forces are considered and articular surfaces are assumed to be rigid. However, extension of this model to study the dynamics of the joint and the inclusion of deformable articular surfaces is also possible.

\subsection{General Description and Assumptions}

Current knee prostheses are designed to have bearing surfaces that are not geometrically congruent [8] for reasons related to human biomechanics and the wear properties of the materials that are used in the prosthetic components. Non-conforming bearing surfaces introduce additional degrees of freedom in knee motion, which further complicates the problem of describing the three-dimensional knee kinematics. One example of an additional degree of freedom is sliding of the femoral component along the bearing surface of the tibial component in a given relative joint angle.

In our knee model, we include patient-specific ligament data to provide the necessary constraints for describing passive kinematics. Each ligament filament is modeled as having a particular length/strain relation, so the passive knee kinematics can be computed by finding the contact state that minimizes the total energy in the ligaments surrounding the knee. That is, each contact state will stretch the ligaments and produce internal strain energy in the filaments that compose the ligament; the contact state that minimizes strain energy is the local equilibrium to which the knee components would relax if disturbed from this equilibrium position.

\section{Methods}

Passive knee kinematics can be described as a series of instantaneous quasi-static solutions to energy minimization [4] of a system in which contact is ideal single-point contact. Potential energy stored in a passive knee is the sum of the energies stored in each filament of the ligaments, because no external load is present. Friction between the rigid articular surfaces is assumed to be negligible.

\subsection{Relative Joint Position and Kinematic Constraints}

The coordinate systems used in this model were similar to those of Blankevoort et al. [2] and Martelli et al. [6]. Two Cartesian coordinate systems were assigned to the major 
bones of the lower limb. The absolute, space-fixed coordinate system was associated with the tibia, and the relative, body-fixed coordinate system was associated with the femur. The $Z$ axes were aligned with the anatomical axis of the limb, with the proximal direction being positive. The $X$ axes were perpendicular to $Z$, lying in the sagittal plane with the anterior direction being the positive $X$ direction. The $Y$ axes were derived as $Y=Z \times X$. Without loss of generality, the origin of the absolute coordinate system was located on the mid-point of the tibial insert in the medial-lateral direction, lying on the resection plane on which the tibial component was fixed. The origin of the relative coordinate system was located on the distal cut for the femoral component, also on the mid-point of the femoral component in the medial-lateral direction. The pose of each prosthetic component was specified with respect to the associated coordinate system.

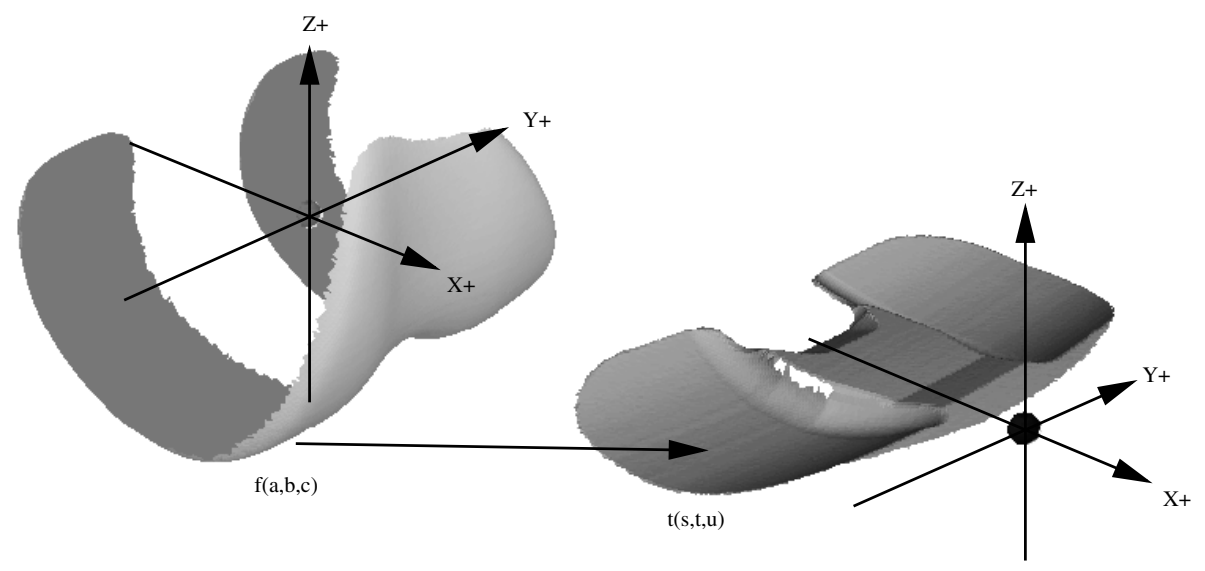

Fig. 1. Two coordinate systems were used. Motion was described as a mobile femur that was rotated and translated with respect to the fixed tibia.

Coordinate systems are related by rigid-body transformations. If $\bar{p}$ is the vector that measures the coordinates of a point in one system, then its coordinates $\bar{q}$ in another system can be expressed as $\bar{q}=T(\bar{p})$ where

$$
\bar{q}=T(\bar{p})=R(\alpha, \beta, \gamma) \bar{p}+\bar{d}
$$

where $R$ is an orthogonal rotation matrix and $\bar{d}$ is a displacement vector. Here, $R$ is described by three successive rotations about the coordinate axes. For angles $\alpha, \beta$, and $\gamma$, abbreviating $\sin (\alpha)=S \alpha, \cos (\alpha)=C \alpha$, and so on, $R(\alpha, \beta, \gamma)=R_{Y}(\beta) R_{Z}(\gamma) R_{X}(\alpha)$ so

$$
R(\alpha, \beta, \gamma)=\left[\begin{array}{ccc}
C \gamma \cdot C \beta & -S \gamma & C \gamma \cdot S \beta \\
C \alpha \cdot S \gamma \cdot C \beta+S \alpha \cdot S \beta & C \alpha \cdot C \gamma & C \alpha \cdot S \gamma \cdot S \beta-S \alpha \cdot C \beta \\
S \alpha \cdot S \gamma \cdot C \beta-C \alpha \cdot S \beta & S \alpha \cdot C \gamma & S \alpha \cdot S \gamma \cdot S \beta
\end{array}\right]
$$

A vector is thus rotated first in flexion (about $Y$ ), then in internal-external rotation (about $Z$ ), then in varus/valgus angulation (about $X$ ), and finally linearly displaced by $\bar{d}$. 
The sequence of rotations was chosen to be flexion angulation first, because flexion is the principal motion in knee kinematics. Internal-external torsion is the next largest angulation, so it was selected as the second angle in the sequence. In practice, varus angulation is very small and, placed as the final angulation, will have little effect on the product of the first two rotations.

\subsection{Surface Contact}

The articular geometry was obtained from 3-dimensional laser surface scanning, in which the inputs were given as a set of points with associated point normals. Assuming that the prosthetic components are ideal rigid bodies, the contact between tibial and femoral articular surfaces is at two contact points, one in the medial compartment and the other in the lateral compartment. Let the contact 4-tuple $\left\{C_{m}, C_{l}, \hat{C}_{m}, \hat{C}_{l}\right\}$ be points on the medial tibial, lateral tibial, medial femoral, and lateral femoral articular surfaces respectively, with corresponding unit normals $\left\{n_{m}, n_{l}, \hat{n}_{m} \hat{n}_{l}\right\}$. There is thus some transformation $T(\cdot)$ such that

$$
\begin{aligned}
\hat{C}_{m} & =T\left(C_{m}\right)=R\left(C_{m}\right)+\bar{d} \\
\hat{C}_{l} & =T\left(C_{l}\right)=R\left(C_{l}\right)+\bar{d} \\
\hat{n}_{m} & =T\left(n_{m}\right)=-R\left(n_{m}\right) \\
\hat{n}_{l} & =T\left(n_{l}\right)=-R\left(n_{l}\right)
\end{aligned}
$$

These equations specify that ideal contact occurs when surface points coincide in space and have local normals pointing in opposite directions.

\subsection{Ligament Strain Energy}

The main ligaments of the knee are the anterior cruciate ligament (ACL), the posterior cruciate ligament (PCL), the medial collateral ligament (MCL), and the lateral collateral ligament (LCL). During the implantation of most total knee prostheses the ACL is resected, so it was eliminated from the model.

Ligaments were modeled as sets of independent, straight filaments. Each filament had a neutral length and stored no strain energy if the distance between the femoral and tibial insertion points was less than or equal to the neutral length. Each filament was modeled as a tension-only linear spring, so the strain energy increased quadratically with its extension and was zero in compression.

Let $L_{i}$ be the instantaneous length of the $i^{\text {th }}$ filament, $\tilde{L}_{i}$ be its neutral length, and $K_{i}$ be its spring constant. The strain energy of the filament was defined as

$$
E_{i}= \begin{cases}K_{i}\left(L_{i}-\tilde{L}_{i}\right)^{2} / \tilde{L}_{i}^{2} & \text { if } L \geq \tilde{L}_{i} \\ 0 & \text { if } L<\tilde{L}_{i}\end{cases}
$$


If $F_{P C L}$ represents the indices of the filaments that constitute the PCL, then the PCL strain energy could be defined as

$$
E_{P C L}=\sum_{i \in F_{P C L}} E_{i}
$$

Defining $E_{A C L}, E_{M C L}$, and $E_{L C L}$ similarly, the governing equation of knee kinematics was to find the contact 4-tuple whose transformation, applied to the femoral insertions of the filaments, minimized the total energy of the system

$$
E_{\text {total }}=E_{P C L}+E_{A C L}+E_{M C L}+E_{L C L}
$$

If a ligament was virtually resected during a simulated surgical procedure the stiffnesses of its filaments $K_{i}$ were adjusted accordingly. For example, if the ligament was resected then all its filament stiffnesses were set to zero.

Equation 3 represents a dimensionless strain energy that is proportional to the ratio of ligament elongation with respect to its neutral length. The energy is normalized to avoid giving bias toward the collateral ligaments, which are naturally much longer than the cruciate ligaments. Because the kinematics are passive, the stiffness constant of the various ligaments are related by scale factors. As recommended in previous work [6], the PCL was given a scale factor of 3.5 times that of the collateral ligaments so that its stiffness was much greater.

\subsection{Computation}

The computation of knee kinematics took place in two stages, referred to as the off-line and online computations. Off-line, the set of all feasible contact 4-tuples were determined for a range of poses. Online, given the insertion locations of a given patient's ligaments and the neutral lengths, the instantaneous contact location that minimized ligament strain energy in each pose was calculated. The kinematics of the knee were then animated as a sequence of instantaneous contact locations.

The advantage of the separation of purely geometrical constraints from a patient's biomechanics was two-fold. First, the off-line computation reduced the real-time requirement in determining the patient-specific kinematics. Second, various surgical strategies, including different prosthetic placements and release of ligaments, could be rapidly simulated by the online computations of the model.

\section{Validation and Results}

To validate our knee model we first created a generic knee model that was symmetric in the sagittal plane. The articular geometry used was size 3 of the Anatomic Modular Knee (AMK) of DePuy Inc. (Warsaw, IN.). The surface geometries were found as sets of points with associated local surface normals. 
Ligament data of a patient were derived from our previous work [6], with ligament insertion locations adjusted so that the MCL was the mirror image of the LCL and posterior cruciate ligament was located at the mid-distance between the collateral ligaments. The PCL, MCL, and LCL were represented by 3, 4, and 4 independent linear springs respectively.

The articular geometries were virtually implanted to a standard position with respect to our ligament data (as recommended by the manufacturer's surgical protocol and subsequently validated by a surgeon). The model was tested in 1,575 distinct angulations. Flexion/extension angles ranged from $0^{\circ}$ to $120^{\circ}$ in $5^{\circ}$ increments. Internal/external rotation angles ranged from $-9^{\circ}$ to $+9^{\circ}$ in $3^{\circ}$ increments. Varus/valgus angle ranged from $-2.0^{\circ}$ to $+2.0^{\circ}$ in $0.5^{\circ}$ increments. At each angulation, the contact state that minimized the total strain energy in the ligaments was noted, as were the individual ligament strains.

\subsection{Ligament Strain and Joint Laxity}

The instantaneous contact location for each pose was determined from the strain energy stored in the posterior cruciate ligament and collateral ligaments. The contribution from each ligament varied through knee flexion. The ligaments states of a knee with standard surgical placement of prosthetic component is plotted in Figure 2.

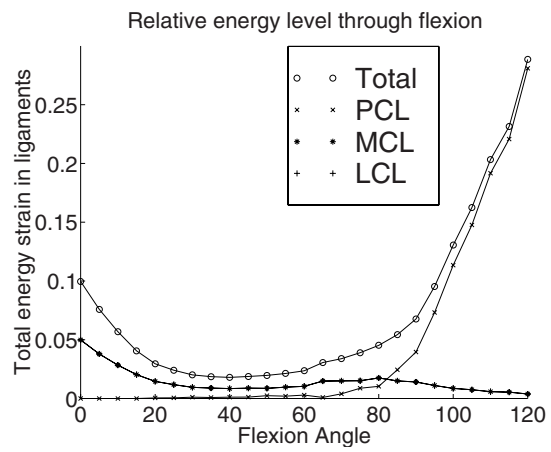

(A) Ligament state

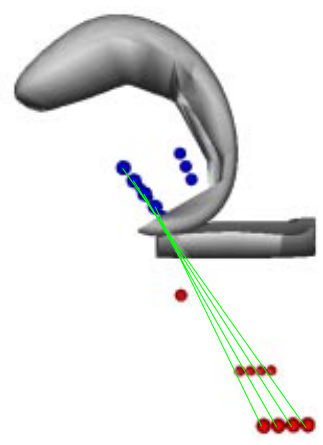

(B) Cross-over of collateral ligaments

Fig. 2. Ligament states of the standard surgical placement. (A) The energy contribution of the ligaments through flexion, the MCL and LCL are identical here. (B) The filaments of the collateral ligament "cross over", as seen in anatomical dissection.

For the standard surgical placement of prosthetic components our model predicted that the PCL was relaxed at low flexion. The collateral ligaments in low flexion contributed the majority of the total ligament strain. As the flexion angle increased the PCL was gradually elongated; its effects on kinematics began at approximately $65^{\circ}$ of knee flexion and in deep flexion the PCL became the predominant constraint of knee motion, which is consistent with anatomical observations. Simultaneously, the collateral ligaments gradually relaxed. At deep flexion the PCL was stretched, causing the femoral 
component to move posteriorly. This prediction is consistent with the results reported by Essinger et al. [4, Page 1234], in which the PCL fibers were shown to be elongated at $120^{\circ}$ of flexion to as much as 1.4 times of the original length.

The range of flexion was limited by PCL strain. Based on previously validated criteria $[5,3]$ and equations presented in the previous section, a numerical value of 0.1 was chosen for the PCL strain that limited knee motion. For standard surgical placement of prosthetic components this corresponds to a range of postoperative motion from $0^{\circ}$ to about $95^{\circ}$ flexion.

The model correctly predicted that cross-over of collateral ligaments occurred at deep flexion, as shown in Figure 2(b).

\subsection{Contact Path and Passive Kinematics}

The contact path on the tibial component is of considerable interest to surgeons and engineers because the wearing characteristics of the polyethylene bearing surface depend on how the surface is loaded over time. Figure 3 depicts the trajectory of the contact path on the tibial bearing surface.

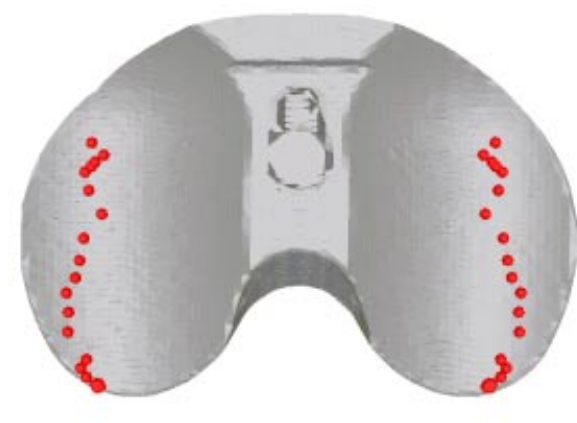

(A) Contact path

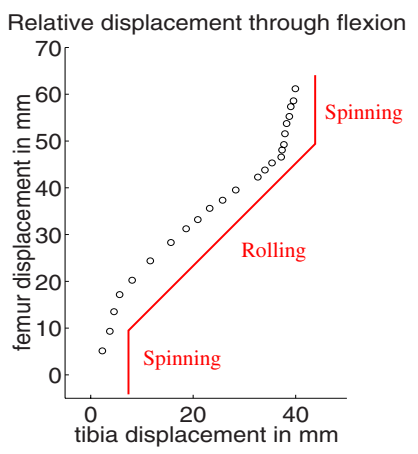

(B) Knee kinematics

Fig. 3. Kinematics of the standard surgical placement. (A) The contact centroid moves posteriorly with increasing flexion. (B) The kinematics proceed from spinning to rolling to spinning with increasing flexion.

For the knee with standard component placement, the tibial contact was located at the anterior center of tibial plateau in full extension. Spinning motion was observed at low flexion angles $\left(<30^{\circ}\right)$. The tibial contact location traveled posteriorly as the flexion angle increased. In mid-flexion the distance between successive contact locations were almost uniform, suggesting that a rolling motion occurred. In deep flexion, the tibial contact locations were located at the posterior aspect of the tibial component. Overall the anterior to posterior movement of the contact locations through flexion/extension angulation corresponded to roll-back motion, which is observed in normal postoperative gait motion. This pattern of motion suggests that polyethylene failure, of the type 
associated with spinning in place, should occur at the extreme anterior and posterior margins of the prosthesis.

\subsection{Alternative Surgical Implantation Strategies}

Both nominal and alternative surgical implantation strategies of the tibial component were simulated. These were anterior and posterior displacement of the component, anterior and posterior sloping of the component, and a combined posterior displacement and posterior sloping. The results are tabulated in Table 1.

Table 1. Comparison of kinematics resulting from different tibial-component placements. Extended/reduced spin was spinning in place for a greater/lesser range of flexion than was observed in the nominal placement.

\begin{tabular}{|l|c|c|c|c|}
\hline \multirow{2}{*}{$\begin{array}{l}\text { Tibial } \\
\text { Placement }\end{array}$} & \multicolumn{3}{|c|}{ Kinematics during Flexion } & \multirow{2}{*}{$\begin{array}{c}\text { Range of } \\
\text { Motion }\end{array}$} \\
\cline { 2 - 4 } & $0^{\circ}-30^{\circ}$ & $30^{\circ}-65^{\circ}$ & $65^{\circ}-120^{\circ}$ & $0^{\circ}-95^{\circ}$ \\
\hline nominal & spin & roll & spin & reduced \\
\hline anterior disp. & roll & roll & extended spin & extended \\
\hline posterior disp. & extended spin & roll & reduced spin & reduced \\
\hline anterior slope & spin & roll & spin & extended \\
\hline posterior slope & spin & roll & extended spin & $\begin{array}{c}\text { greatly } \\
\text { extended }\end{array}$ \\
\hline $\begin{array}{l}\text { posterior disp } \\
\& \text { slope }\end{array}$ & spin & roll & spin & \\
\hline
\end{tabular}

The knee with an anteriorly displaced tibial component had different knee kinematics than the standard knee. Predicted kinematics for low flexion angles was a rolling motion instead of a spinning motion. The range of motion was also reduced. This was because anteriorly displacement of tibial component effectively increased the distance between femoral and tibial PCL insertion sites, which led to a highly elongated PCL at high flexion. Similarly, an anteriorly sloped tibial component also had reduced range motion because anterior sloping increased the distance between ligament insertion sites.

Posterior displacement or sloping of the tibial component increased the range of motion, in part because such placements of tibia decreased the distance between PCL insertion sites. Of particular interest is the knee with both posteriorly displaced and posteriorly sloped tibial component; it had greatly increased range motion and the overall kinematics were still a spin-roll-spin motion.

\subsection{Applications}

Because of the separation of on-line computation from off-line computation, knee kinematics from full extention to deep flexion can be calculated in just a few minutes from any specific ligament configuration. This facilitates potential applications of the knee model, including: 
Preoperative surgical planning: If data of a patient's knee can be obtained prior to surgery, the surgeon can preoperatively simulate the surgical procedure. This planning can include determination of the most suitable prosthesis design and appropriate surgical placement for the patient. Effects of different sizes of components and various sloping of the tibial insert can also be visualized.

Intraoperative surgical adjustment: The simulation can run fast enough that the surgeon can intraoperatively simulate modifications to a preoperative plan. For example, effects of ligament release and of alternate sloping of the tibial component can be simulated before the surgeon actually performs the tissue modifications.

Prosthesis Design: Current knee prosthesis are designed to have non-congruent bearing surfaces. The effect of different design parameters, such as the flatness of the tibial insert and radii of the femoral head, can be simulated for placement in a "standard" patient or in a suite of actual patients for whom knee data are available. The simulations can be used directly, or can act as input into a subsequent statistical model of how knee design parameters affect knee motion.

\section{Conclusions}

We have described the development and construction of a computational model of the kinematics of condylar-type total-knee prostheses. The model required knowledge of the geometry of articular surfaces, the insertion locations and relative mechanical properties of the knee ligaments, and the implanted location of the prosthetic components. Based on the principle of ligament strain minimization $[4,6]$, the computer program generated the motion of the center of contact of each condyle, the kinematics of the prosthetic joint, and the resulting ligament state from full extension to deep flexion.

The model was first validated on a prosthesis design for which the lateral profiles were is the mirror images of the medial profiles. Using the articular surfaces of an AMK prosthesis and ligament data derived from previous work [6], the model was used to simulate the spatial kinematics of a prosthetic knee. The model demonstrated that the pattern of knee motion was influenced by the design of the prosthesis and by the surgical implantation strategies of the prosthetic components. With respect to the AMK design, the simulation suggested that spinning and point-loading motions take place with a wide variety of prosthesis implantation strategies.

The model provided a unique tool for evaluating the effects of different surgical implantation strategies. The model predicted that posterior displacement or sloping of the tibial component would usually lead to improved patterns of knee kinematics and increased postoperative ranges of motion. Conversely, anterior displacement or sloping of the tibia would have adverse effects, such as excessive elongation of the PCL and point loading in deep flexion. These finding are consistent with current knowledge of the in vivo behavior of this prosthesis design $[5,9]$. 
The model was validated by simulating a simplified geometry of the knee of an actual patient. The model predictions were be plausible and in accordance with published results. Further validation by in vitro and in vivo experiments are needed, particularly to consider the effects of asymmetric ligaments and asymmetric components. The model may prove useful not only to determine patient-specific implantation parameters but also to design new knee components that interact better with the biological structures of the human knee.

\section{Acknowledgements}

This research was supported in part by Communications and Information Technology Ontario, the Institute for Robotics and Intelligent Systems, and the Natural Sciences and Engineering Research Council of Canada.

\section{References}

[1] L. Blankevoort, R. Huiskes, and A. de Lange. The envelope of passive knee joint motion. Journal of Biomechanics, 21(9):705-720, 1988.

[2] L. Blankevoort, J. H. Kuiper, R. Huiskes, and H. J. Grootenboer. Articular contact in a three-dimensional model of the knee. Journal of Biomechanics, 24(11):1019-1031, 1991.

[3] D. L. Butler, M. D. Kay, and D. C. Stouffer. Comparison of material properties in fasciclebone units from human patellar tendon and knee ligaments. Journal of Biomechanics, 19(6):425-432, 1986.

[4] J. R. Essinger, P. F. Leyvraz, J. H. Heegard, and D. D. Robertson. A mathematical model for the evaluation of the behaviour during flexion of condylar-type knee prostheses. Journal of Biomechanics, 22(11-12):1229-1241, 1989.

[5] A. Garg and P. S. Walker. Prediction of total knee motion using a three-dimensional computer-graphics model. Journal of Biomechanics, 23(1), 1990.

[6] S. Martelli, R. E. Ellis, M. Marcacci, and S. Zaffagnini. Total knee arthroplasty kinematics, computer simulation and intraoperative evaluation. The Journal of Arthroplasty, 13(2):145155, February 1998.

[7] T. J. A. Mommersteeg, R. Huiskes, L. Blankevoort, J. G. M. Kooloos, J. M. G. Kauer, and P. G. M. Maathuis. A global verification study of a quasi-static knee model with multibundle ligaments. Journal of Biomechanics, 29(12):1659-1664, 1996.

[8] J. J. O'Connor and J. W. Goodfellow. The role of meniscal bearing vs. fixed interface in unicondylar and bicondylar arthroplasty. In V. M. Goldberg, editor, Controversis of Total Knee Arthroplasty, pages 27-49. Raven Press, 1991.

[9] L. A. Whiteside and D. D. Amador. The effect of posterior tibial slope on knee stability after Ortholoc total knee arthroplasty. Journal of Arthroplasty, 3(Supplement):S51-S57, October 1988.

[10] J. Wismans, F. Veldpaus, J. Janssen, A. Huson, and P. Struben. A three-dimensional mathematical model of the knee-joint. Journal of Biomechanics, 13(8):677-685, 1980. 